\title{
Temperature Effect on Breakdown Performance of Insulating Polymer Thin Films
}

\author{
M. Ritamäki, I. Rytöluoto, K. Lahti \\ Department of Electrical Engineering \\ Tampere University of Technology, Tampere, Finland
}

\begin{abstract}
The effect of temperature on the breakdown performance of metallized capacitor-grade BOPP film was characterized using a large-area multi-breakdown measurement method. The measurements conducted in the temperature range from room temperature to $100{ }^{\circ} \mathrm{C}$ revealed a linearly decreasing trend in the characteristic $63.2 \%$ breakdown probability after $60{ }^{\circ} \mathrm{C}$ and the presence of a smaller weak spot subpopulation. The weak spots remained relatively unaffected by temperature, and first breakdowns occurred in a seemingly statistical manner. The brief exposure to elevated temperature was considered as one explanation behind the variations in the number of weak points, but fluctuations in film quality and Weibull dynamics were seen are more probable explanations. The results demonstrate the possibility of multiple breakdown mechanisms and their different temperature-dependency, and demonstrate the high short-term breakdown strength of BOPP film even at high temperatures.
\end{abstract}

\section{Introduction}

Recently there has been a growing demand for capacitor dielectrics for high-temperature applications. Biaxially oriented polypropylene (BOPP) has been used in high power density capacitors, with metallized electrodes providing self-healing capabilities and graceful aging behavior [1]. When compared to traditional temperature-resistant plastics such as polytetrafluoroethylene (PTFE), poly(phenylene sulfide) (PPS) or polyimides polypropylene offers high dielectric breakdown strength, good self-healing capabilities and polypropylene films can be manufactured to thicknesses in the range of few $\mu \mathrm{m}$, facilitating its use in low and mediumvoltage applications [2]. Polypropylene melts in the region of $165-170{ }^{\circ} \mathrm{C}$, but its operating temperature has been limited to $85^{\circ} \mathrm{C}$, or with voltage deration to about $105^{\circ} \mathrm{C}$. [2]

The maximum operating temperature of polymer dielectrics is largely determined by the increasing losses and decreasing dielectric breakdown strength at higher temperatures. In [3] and [4] the effect of temperature on the breakdown strength of polymer materials has been divided in low and high temperature regions. In the low temperature region the dielectric breakdown strength is relatively independent of the temperature, whereas in the high temperature region the breakdown strength decreases with temperature. For polar polymers a "critical temperature" may exist, above which the breakdown strength falls rapidly. In [3] a decreasing electric strength with increasing temperature has been reported for isotactic polypropylene. Owing to its nonpolar nature there is no critical temperature. Contradicting views are presented in [5] in which it is presented that the dielectric breakdown strength of polymers is unaffected by temperature.

Capacitor-grade films differ from traditional polymer materials, especially in terms of the biaxial orientation and the purity of the base polymer. In this paper the effect of temperature on the breakdown behavior of commercial capacitor-grade $\mathrm{Zn} / \mathrm{Al}$-metallized PP film is evaluated from room temperature to $100{ }^{\circ} \mathrm{C}$. A largearea multi-breakdown measurement method detailed in [6], [7] was utilized. By taking advantage of the selfhealing capabilities of metallized PP film total sample areas up to $350 \mathrm{~cm}^{2}$ were measured at each temperature step. Large measurement areas allow measuring statistically significant amount of breakdowns even from the lower-probability regions.

\section{Experimental}

\subsection{Material and sample details}

A commercial zinc/aluminum alloy metallized capacitor-grade BOPP film manufactured by Tervakoski Films Group was used in the experiment. The film had a manufacturer-reported thickness of $6 \mu \mathrm{m}$ and the one-sided metallization had a main body surface resistance of 5$12 \Omega / \square$ and a reinforced edge with a surface resistance of $2-4 \Omega / \square$. The $72.5 \mathrm{~mm}$ wide film had a free margin $2.5 \mathrm{~mm}$. Six $110 \mathrm{~mm}$ samples plus an additional six reference samples were prepared for each temperature, with the exception of the room temperature measurement for which no reference samples were needed.

\subsection{Measurement setup}

Measurements at elevated temperature were conducted in atmospheric air by utilizing a test oven detailed in [8]. The oven temperature was controlled by a selftuning PID controller and a cross-flow fan was utilized to promote air circulation. The test fixture was placed in the oven and its temperature was let to stabilize for 1015 minutes. Both the air and fixture temperatures were 
monitored during this stabilization period, with the fixture temperature monitored by a K-type thermocouple placed between the bench edge and the top plate. The oven was opened to remove the thermocouple before the multi-breakdown measurements. Between the removal of the thermocouple and the actual measurement the system was let to stabilize for approximately 1-2 minutes.

To determine the reversibility of the measured changes for each high temperature measurement one reference sample in a paper envelope was placed in the upper section of the oven. The reference sample was removed after the multi-breakdown measurement and measured after a few days, having had time to settle at room temperature.

Six film samples were measured at room temperature and at temperatures of 60,80 and $100{ }^{\circ} \mathrm{C}$. Excessive film curling resulted in the loss of one reference sample for each temperature; therefore the reference measurements consisted of five samples each.

\subsection{Large-area multi-breakdown measurement}

A large area multi-breakdown measurement detailed in [6], [7] was applied to determine changes in the breakdown behavior. The self-healing test capacitors were manufactured by placing two $90 \mathrm{~mm}$ wide $12 \mu \mathrm{m} \mathrm{Zn/Al}$ metallized BOPP films (main body metallization resistivity $5-12 \Omega / \square$ ) on both sides of the test sample. The one-sided metallization of the test sample functioned as the lower electrode and the $12 \mu \mathrm{m}$ film was used to make electrical contact between it and the test fixture. The upper $12 \mu \mathrm{m}$ film functioned as the other electrode. This arrangement resulted in an active area of approximately $58 \mathrm{~cm}^{2}$, with six samples equaling to a total measured area of approximately $350 \mathrm{~cm}^{2}$. To provide mechanical support two $100 \mu \mathrm{m}$ thick polyester films were placed on both sides of the film stack. The film stack was attached to a test fixture consisting of a Bakelite baseboard and two glass plates. The $12 \mu \mathrm{m}$ metallized films were clamped between two aluminum plates on opposing edges of the fixture, to which the electrical connections were made. The test capacitor arrangement is illustrated in Figure 1.

Loosely according to IEC-60243-1 slow rate-of-rise test [9] the measurement consisted of a fast voltage ramp up at $400 \mathrm{~V} / \mathrm{s}$ to $1800 \mathrm{~V}$, corresponding to a field of $300 \mathrm{~V} / \mu \mathrm{m}$, followed by a slow ramp at $30 \mathrm{~V} / \mathrm{s}$. Breakdowns were measured during the slow ramp which was continued until no more discharges were detected.

Based on an equation presented in [10] the $2.5 \mathrm{~mm}$ free margin was presumed to exhibit flashovers above 4-5 kV. The inception voltage for repeating flashovers was determined using an otherwise similar $9 \mu \mathrm{m}$ metallized film, in which rapid flashovers were measured at DC voltages above $5.5-6 \mathrm{kV}$. For the $6 \mu \mathrm{m}$ film flashovers across the free margin were not seen as a limiting issue since the test voltage rarely exceeded $5 \mathrm{kV}$.
The measurement used a Spellman SL1200 DC high voltage supply, controlled and monitored using a resistive Spellman 1:10 000 voltage divider, National Instruments PCI-6221 DAQ card and a LabVIEW-based program. Test capacitor voltage and circuit current were monitored real-time using one AC and one DC coupled Tektronix P6015 high voltage probe and a $1.0330 \Omega$ impulse current measurement resistor. Discharge current and voltage waveforms were captured by a LeCroy HRO 66Zi oscilloscope set to trigger at the falling edge of the AC coupled voltage channel. Compared to the current triggering method in [6], [7] the voltage drop based trigger enabled detection of discharges with substantially lower energies. An USB-camera was used to provide a video feed from above the test fixture which was monitored to verify the absence of repeating flashovers at the sample edges.

A two-criterion breakdown selection procedure detailed in [6] was used to exclude non-independent breakdowns and non-breakdown discharges from the measurement data. These included phenomena such as multiple sequential self-healings occurring rapidly in one spot, breakdowns at previously cleared areas, surface flashovers and other non-breakdown discharges. To uniform the results and due to the increased probability of surface flashovers at the free margin near the end of the measurements for each sample any discharges beyond the initial 50 were omitted from further analysis.

\subsection{Statistical analysis}

Weibull statistical analysis was used to fit the breakdown data with suitable distributions to reach a visually and mathematically adequate fit. In every measurement deviation from a single 2-parameter Weibull distribution was evident and additively mixed two-subpopulations were used [6], [7], [11]:

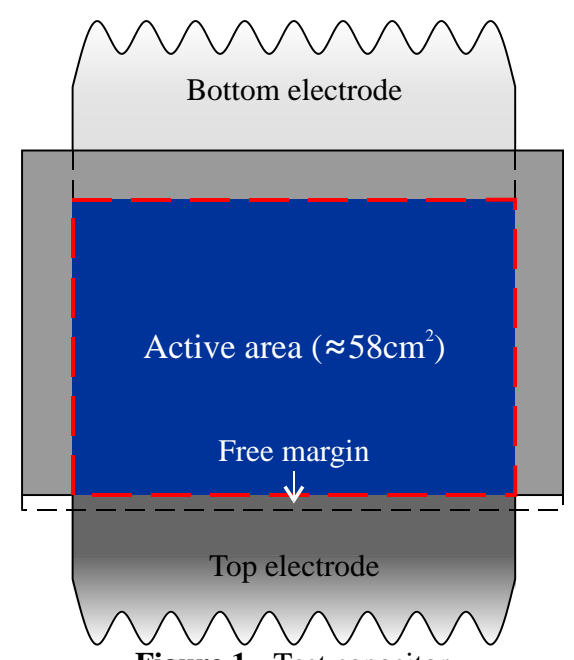

Figure 1 - Test capacitor 
Table 1 - Weibull parameters

\begin{tabular}{cccccccc} 
& $\mathrm{RT}$ & $60{ }^{\circ} \mathrm{C}$ & $80{ }^{\circ} \mathrm{C}$ & $100{ }^{\circ} \mathrm{C}$ & $\mathrm{REF}-60{ }^{\circ} \mathrm{C}$ & $\mathrm{REF}-80{ }^{\circ} \mathrm{C}$ & $\mathrm{REF}-100{ }^{\circ} \mathrm{C}$ \\
\hline Number of points & 90 & 119 & 93 & 88 & 79 & 104 & 97 \\
Weibull $\beta_{1}$ & 2.51 & 3.67 & 5.86 & 5.71 & 4.04 & 4.69 & 7.14 \\
Weibull $\alpha_{1}(\mathrm{~V} / \mu \mathrm{mm})$ & 507 & 468 & 527 & 506 & 516 & 570 & 601 \\
$N_{1} / N$ & 0.08 & 0.12 & 0.19 & 0.24 & 0.25 & 0.29 & 0.41 \\
Weibull $\beta_{2}$ & 23.72 & 16.69 & 19.76 & 20.48 & 24.94 & 27.88 & 23.80 \\
Weibull $\alpha_{2}(\mathrm{~V} / \mu \mathrm{mm})$ & 686 & 665 & 644 & 610 & 686 & 694 & 698 \\
$N_{1} / N$ & 0.92 & 0.88 & 0.81 & 0.76 & 0.75 & 0.71 & 0.59
\end{tabular}

$$
\begin{aligned}
& F(x)=\frac{N_{1}}{N}\left[1-\exp \left\{-\left(\frac{x}{\alpha_{1}}\right)^{\beta_{1}}\right\}\right]+\ldots \\
& \ldots \frac{N_{2}}{N}\left[1-\exp \left\{-\left(\frac{x}{\alpha_{2}}\right)^{\beta_{2}}\right\}\right]
\end{aligned}
$$

In (1) $F(x)$ is the 2-parameter two-subpopulation Weibull distribution, $N_{1} / N$ and $N_{2} / N$ are the relative portions of and $\beta$ and $\alpha$ are the Weibull scale and shape parameters of the two subpopulations. The scale parameter $\alpha$ represents the $1-\mathrm{e}^{-1} \approx 63.2 \%$ breakdown probability and the shape parameter $\beta$ depicts the homogeneity of the data points, being analogous to the inverse of the standard deviation of Gaussian distributions. The first subpopulation primarily accounts for the low field breakdowns whereas the second one represents the intrinsic behavior, which in this paper refers to the region of recurring breakdowns. The distribution parameters were estimated with maximum likelihood estimation (MLE) or non-linear rank regression (NLRR) methods. One-sided $90 \%$ confidence bounds were calculated using Fisher Matrix (FM) -based method. Calculations were done using Weibull++ 9 software.

Given the nature of the multi-breakdown measure-

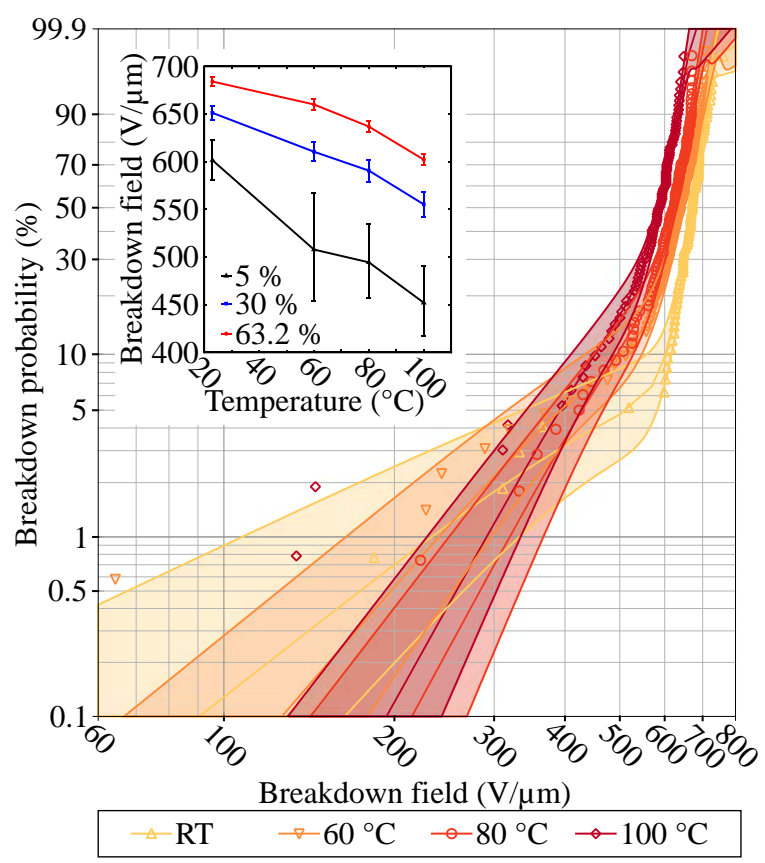

ment unequal number of breakdowns was recorded in every measurement. Despite this the amount of selected breakdowns was around 90 for each temperature step, and more importantly the trend in the amount of selected points does not correlate with the changes observed in the breakdown behavior. Therefore the error caused by the variation between the numbers of points was seen insignificant at least in the $>10 \%$ probability region. The increasing uncertainty in the low probability region is evident from the broadening confidence bounds.

\section{Results and discussion}

The distribution parameters and the number of selected breakdowns are presented in Table 1 . Data points and Weibull distributions from measurements at elevated temperature are presented in Figure 2a together with percentiles corresponding to $5 \%, 30 \%$ and $63.2 \%$ breakdown probabilities. Both are presented with onesided $90 \%$ confidence bounds. Data points and distributions from corresponding reference measurements at room temperature are presented in Figure 2b. Looking at the results it can be summarized that:

- the characteristic $63.2 \%$ breakdown strength

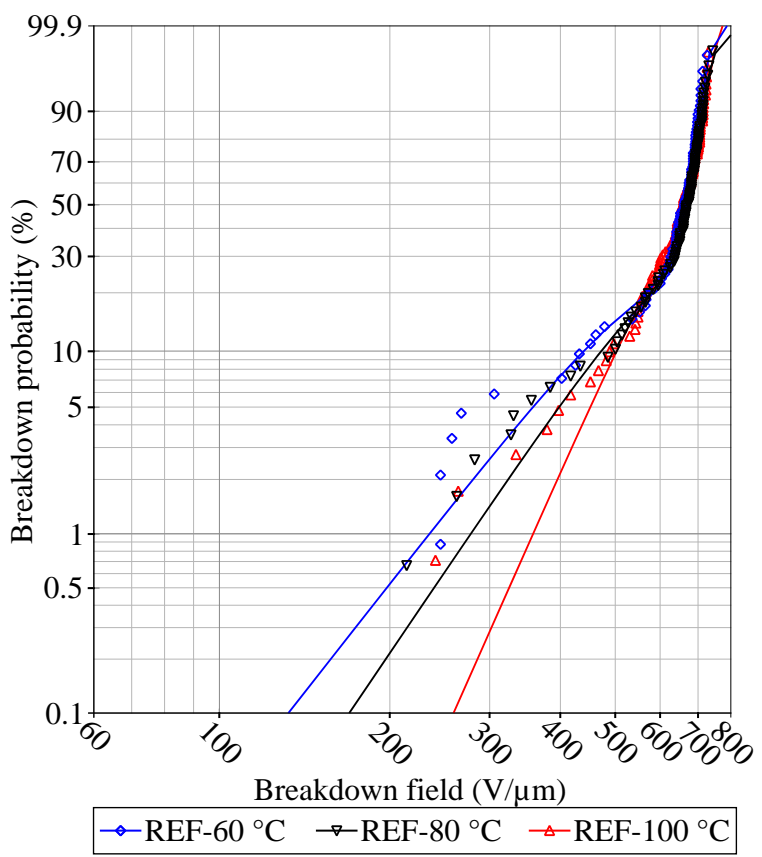

Figure 2 - a) Breakdown behavior at room temperature, 60,80 and $100{ }^{\circ} \mathrm{C}$ together with calculated $5 \%$, $30 \%$ and $63.2 \%$ percentiles b) Reference measurements at room temperature 
decreased from $684 \mathrm{~V} / \mu \mathrm{m}$ at room temperature to $602 \mathrm{~V} / \mu \mathrm{m}$ at $100{ }^{\circ} \mathrm{C}$

- the decrease can be considered as reversible, since the reference samples displayed similar behavior at room temperature

- two subpopulations were distinguishable in all measurements

- $\quad$ the effect of temperature was most significant in the second Weibull subpopulation

- the weak spot subpopulation was not heavily influenced by the elevated temperature

In [7] similar multi-breakdown measurement was used to characterize areas up to $1950 \mathrm{~cm}^{2}$ of same brand of film from different roll at room temperatures. The distinct weak spot subpopulation and the intrinsic region reported here correspond to the first two portions of the S-shape [7], and the absence of the third subpopulation can be explained by the omission of any breakdowns beyond the initial 50. Additionally the Weibull parameters of the first two subpopulations are fully in line with presented in Table 1 . This supports the repeatability of the measurement method.

The shape of the Weibull distribution remains relatively unchanged with temperature. Moreover based on [6] it is presumed that the variations in Weibull $\beta_{2}$ are at least partially due to the use of average thicknesses for breakdown field determination.

Given the minor variations in the distribution shape the decreasing trends in the $30 \%$ and $63.2 \%$ percentiles and in the Weibull $\alpha_{2}$ are similar. Although a similar trend is also observed in the $5 \%$ percentiles, the statistical uncertainty, as evident in the large error bars, limits the usability of the data. The visually discernible inverse linear trend in data after $60{ }^{\circ} \mathrm{C}-100{ }^{\circ} \mathrm{C}$ is in line with the results presented for isotactic polypropylene in [3], in which a similar decrease is reported approximately after $50-60{ }^{\circ} \mathrm{C}$. The room temperature measurements are omitted and based on the data from $60-100{ }^{\circ} \mathrm{C}$ an inverse linear relationship between the Weibull $\alpha_{2}$ and the temperature $\mathrm{T}$ is formulated as:

$$
\alpha_{2}=k T+b
$$

where $k$ and $b$ are constants with values of -1.38 and $749.9 \mathrm{~V} / \mu \mathrm{m}$ respectively. The fit was calculated using MATLAB software and has a R-square value of 0.9843 . Based on charts presented in [3] the relationship is presumed hold from $60^{\circ} \mathrm{C}$ up to $130{ }^{\circ} \mathrm{C}$, but additional research is needed to confirm validity especially above $100{ }^{\circ} \mathrm{C}$.

For practical applications however the presence of a distinct weak spot subpopulation is of more importance than the changes in the high field behavior. Given typical operating fields below $100 \mathrm{~V} / \mu \mathrm{m}$ even for high energy density metallized film designs, the temperature of $100{ }^{\circ} \mathrm{C}$ does not result in the operating field exceeding the intrinsic breakdown strength.

The presence of two subpopulations may indicate the presence of two breakdown mechanisms [5], out of which the mechanism responsible for low field breakdowns is not heavily affected by temperature. Accurate determination of its temperature-dependency is made impossible by the scarcity of data points in the low probability region. However the nature of the two proposed breakdown mechanisms cannot be determined using the available data.

In addition to the large-area multi-breakdown results published for same brand of film in [7], in related literature similar two-subpopulation breakdown behavior has been reported as fingerprint-like in unaged PP film [12] and as a result from thermoelectric [13] and thermal aging [8] of PP film having previously displayed single population breakdown behavior. The aging cycles after which diverging weak spots were measured spanned from 20 hours of thermoelectric aging [13] to 144 hours of thermal aging [8]. Even though in this study the films were exposed to elevated temperature for a relatively short period of 10-20 minutes fewer low field breakdowns were measured in room temperature measurement. It is therefore presented that

- $\quad$ at least a portion of the low field breakdowns are a part of the characteristic "fingerprint" of the film

- the exposure to elevated temperature may have caused material degradation leading to more low field breakdowns.

In the reference films the relative portion of the first subpopulation increased from 0.25 after $60{ }^{\circ} \mathrm{C}$ to 0.41 after $100{ }^{\circ} \mathrm{C}$, but this increase was simultaneous with increasing Weibull $\alpha_{1}$, suggesting that the increase is related to Weibull dynamics rather than actual breakdown phenomena. Additionally the distributions in Figure $2 b$ are in line with each other. Therefore it is considered more probable that the low field breakdowns were solely an intrinsic film property, and that the variations were a result from the statistical nature of breakdown phenomena and fluctuations in film quality rather than from rapid heat-induced degradation. If the low field breakdowns are regarded solely as an intrinsic material property is should be noted the first few breakdowns occurred in a seemingly statistical manner independent of temperature.

Given the lack of temperature dependency in the low probability region, based on [2] dielectric losses are established as the main limiting factor for use at higher temperatures, requiring voltage deration. In addition to intolerable power loss rapidly increasing losses may lead to thermal runaway-type breakdown phenomena. Nevertheless in the multi-breakdown measurement selfhealing phenomena worked successfully up to $100{ }^{\circ} \mathrm{C}$, and no permanent insulation failures were observed. It is however worth noting that the measurement duration of few minutes is not long enough to rule out the possibility of breakdown and degradation mechanisms acting in time scales from minutes to hours. If such mechanisms exist they may also limit the usability of high temperatures. Therefore broad characterization of dielectric and aging properties is needed before any materi- 
als can be regarded sufficiently stable for long-term use at elevated temperatures.

\section{Conclusions}

Large-area multi-breakdown measurements using metallized capacitor-grade BOPP film revealed the presence of temperature-independent weak spots, and twosubpopulation Weibull distributions were applied to reach a visually and mathematically good fit. An inverse power law relationship between temperature and Weibull $\alpha_{2}$ was determined between $60-100{ }^{\circ} \mathrm{C}$. Similar trend was also evident in $30 \%$ and in the characteristic $63.2 \%$ breakdown strengths.

Diminishing differences were measured in reference samples measured at room temperature after equivalent thermal stresses, based on which the changes in the intrinsic behavior were regarded as a reversible effect of temperature rather than heat-induced degradation. Compared to the room temperature reference the relative portion of the weak spot subpopulation increased in all films subjected to elevated temperature. For this heatinduced degradation and fluctuations in film quality were proposed as complementary explanations, out of which the latter was considered more probable.

\section{References}

[1] W. J. Sarjeant, "Capacitor fundamentals," in Electrical Electronics Insulation Conference, 1989. Chicago '89 EEIC/ICWA Exposition., Proceedings of the 19th, 1989, pp. 151.

[2] J. Ho and R. Jow, "Characterization of High Temperature Polymer Thin Films for Power Conditioning Capacitors," Adelphi, MD 20783-1197, 2009.

[3] M. Ieda, "Dielectric Breakdown Process of Polymers," Electr. Insul. IEEE Trans., vol. EI-15, no. 3, pp. 206-224, 1980.

[4] A. K. Jonscher and R. Lacoste, "On a Cumulative Model of Dielectric Breakdown in Solids,” Electr. Insul. IEEE Trans., vol. EI-19, no. 6, pp. 567-577, 1984.

[5] L. Dissado and J. C. Fothergill, Electrical degradation and breakdown in polymers. Peter Peregrinus Ltd., 1992.

[6] I. Rytöluoto, K. Lahti, M. Karttunen, and M. Koponen, "LargeArea Dielectric Breakdown Performance of Polymer Films Part I: Measurement Method Evaluation and Statistical Considerations on Area-dependence,” IEEE Trans. Dielectr. Electr. Insul., vol. 22, no. 2, pp. 689-700, 2015.

[7] I. Rytoluoto and K. Lahti, "New approach to evaluate areadependent breakdown characteristics of dielectric polymer films,” Dielectr. Electr. Insul. IEEE Trans., vol. 20, no. 3, pp. 937-946, 2013.

[8] M. Ritamäki, "Effects of Thermal Aging on Polymer Thin Film Insulations for Capacitor Applications,” Tampere University of Technology, 2014.

[9] IEC, "International standard IEC 60243-1, Part 1: Tests at power frequencies. Edition 3.0.” 2013.

[10] V. Belko, O. Emelyanov, and M. Shemet, "A study of nanosecond arcsurface flashover in metallized polymer films," in Electrical Insulation and Dielectric Phenomena (CEIDP), 2013 IEEE Conference on, 2013, pp. 476-477.

[11] W. Hauschild and W. Moss, Statistic Techniques for High Voltage Engineering. London: Peter Peregrinus Ltd., 1992.

[12] S. J. Laihonen, "Polypropylene: Morphology, defects and electrical breakdown,” Kungliga Tekniska Högskolan, 2005.

[13] B. Gosse, J. P. Gosse, S. Saïd, A. Gadoum, and M. Nemamcha, "Electrical degradation of polypropylene: A study by FTIR microspectroscopy,” J. Appl. Polym. Sci., vol. 46, no. 6, pp. 1121-1124, Oct. 1992. 